\title{
Avaliação do Centro de Referência em Saúde do Trabalhador Cearense pelos Usuários e Representante dos Sindicatos
}

\author{
Suz̨ete Rodrigues Leônidas - Universidade de Fortalez̧a, Fortaleza, Brasil \\ Cynthia de Freitas Melo - Universidade de Fortaleza, Fortaleza, Brasil
}

\begin{abstract}
Resumo
Este estudo teve como objetivo avaliar o Centro de Referência em Saúde do Trabalhador (CEREST) estadual do Ceará a partir das crenças dos representantes de sindicatos e usuários. Para tanto, foi realizada pesquisa de campo, de cunho qualitativo, aplicada, do tipo exploratória e descritiva. Contou com a participação de três representantes de sindicatos e cinco usuários que responderam a uma entrevista semiestruturada. As informações foram submetidas à análise de conteúdo de Bardin e à análise de dados textuais com apoio do software Interface de $\mathrm{R}$ pour les Analyses Multidimensionnelles de Textes et de Questionnaires (IRAMUTEQ). Os resultados apontaram entraves na gestão e na falta de clareza das funções do CEREST e a falta de profissionais. Conclui-se que existe uma defasagem entre trabalho prescrito e trabalho real causado por entraves políticos e problemas de gestão que acabam distanciando as ações do CEREST no Ceará do seu objetivo de prevenção e promoção da saúde do trabalhador. Palavras-chave: saúde, programas sociais, trabalhador, sindicato
\end{abstract}

\section{Reference Center Evaluation of Health Worker Cearense by Users and Trade Union Representative}

\begin{abstract}
This study aimed to evaluate the Ceará State Occupational Health Reference Center (CEREST) based on the beliefs of union representatives and users. For this purpose a qualitative descriptive-exploratory applied field research was conducted. Participants included three representatives of trade unions and five users who answered a semi-structured interview. The information was submitted to Bardin content analysis and analysis of textual data with support of the software Interface de $\mathrm{R}$ pour les Analyses Multidimensionnelles de Textes et de Questionnaires (IRAMUTEQ). The results showed barriers in the management and lack of clarity of CEREST's functions as well as lack of professionals. We conclude that there is a gap between prescribed and real work due to political obstacles and management problems that end up distancing CEREST's actions from its goal of prevention and promotion of workers' health.

Keywords: health; social programs; worker; trade union
\end{abstract}

\section{Referencia del Centro de Evaluación de la Salud del Trabajador por Usuarios Cearense} y Representante de los Sindicatos

\begin{abstract}
Resumen
Este estudio tuvo como objetivo evaluar el Centro de Referencia en Salud del Trabajador (CEREST) estadual de Ceará a partir de las creencias de los representantes sindicales y los usuarios. Por lo tanto, fue realizada una investigación de campo, de naturaleza cualitativa, aplicada, de tipo exploratoria y descriptiva. Contó con la participación de tres representantes de los sindicatos y cinco usuarios que respondieron a una entrevista semiestructurada. Las informaciones fueron sometidas a análisis de contenido de Bardin y análisis de datos textuales con apoyo del software de Interface pour les Analyses Multidimensionnelles de Textes et de Questionnaires (IRAMUTEQ). Los resultados señalan obstáculos en la gestión, y en la falta de claridad de las funciones del Cerest y la falta de profesionales. Llegamos a la conclusión de que existe um desfazaje entre el trabajo prescripto y el trabajo real causado por obstáculos políticos y problemas de gestión que terminan distanciando las acciones del CEREST en Ceará de su objetivo de prevención y promoción de la salud del trabajador.

Palabras-clave: salud; programas sociales; trabajador; sindicato
\end{abstract}

\section{Introdução}

O contexto econômico, social e político do fim da década de 1970 e início dos anos de 1980 no Brasil, acompanhado pela conjuntura da Reforma Sanitária, foi decisivo para elaboração do projeto de reestruturação das políticas de saúde no país (Lourenço \& Lacaz, 2013). A partir da Constituição Federal de 1988, da criação e consolidação do Sistema Único de Saúde (SUS) e da Lei Orgânica da Saúde 8.080/90, o setor saúde do estado passa a coordenar as ações de Saúde do Trabalhador, intervindo nos espaços de trabalho (Costa, Lacaz, Jackson Filho, \& Vilela, 2013; Medeiros, Sarleno, Silvestre, \& Magalhães, 2103).

Entre os projetos para implementar a Política de Saúde do Trabalhador destaca-se a implantação da Rede Nacional de Atenção Integral à Saúde do Trabalhador (RENAST) a partir da Portaria do Ministério da Saúde de $n^{0} 1.679$ (2002). A RENAST constitui-se como uma rede de cobertura nacional e tem como objetivo a criação dos Centros de Referência em Saúde do Trabalhador (CERESTs) e como instrumento estratégico 
a difusão dos princípios e práticas do campo da Saúde do Trabalhador no SUS, em todos os níveis de atenção (Dias \& Silva, 2013; Leão \& Castro, 2013; Ministério da Saúde [MS], 2009; Quilião, Fassa, \& Restrepo, 2013).

Os CERESTs são retaguardas técnicas e científicas junto aos profissionais da rede. Suas atribuições compreendem a fiscalização das condições de trabalho e vigilância em saúde do trabalhador, avaliação e diagnóstico de ambiente laboral, realização de projetos específicos a partir das reinvindicações dos sindicatos e das solicitações da rede de saúde, programas de capacitação, orientação e educação em saúde e atendimento ambulatorial (Balista, Santiago, \& Corrêa Filho, 2011; Daldon, 2012). A instituição dos CERESTs surge para intervir de forma integrada e articulada em todos os níveis de gestão do sistema como a área de planejamento e de regionalização, os polos de educação permanente, a atenção básica, ambulatorial, hospitalar e a vigilância em saúde e ambiental (Jacques, Milanez, \& Mattos, 2012; Lacaz, Trapé, Soares, \& Santos, 2013; MS, 2012).

Atualmente, no país, são cerca de 190 CERESTs habilitados. No estado do Ceará, são oito CERESTs regionais e um estadual, sendo este último o foco do presente trabalho. O Centro Estadual de Referência em Saúde do Trabalhador Manoel Jacaré (conhecido como CEREST-CE) é formado por uma equipe multidisciplinar composta atualmente por médico do trabalho, enfermeiro, técnico de segurança do trabalho, fisioterapeuta, fonoaudiólogo e assistente social (Jacques et al., 2012; Oliveira, 2013). O CEREST-CE compartilha o desafio de garantir a viabilidade e a sustentabilidade social, política e econômica do SUS no Ceará (Machado et al., 2013). Devido às suas peculiaridades regionais e dificuldades de operacionalização, presente nos diversos programas do SUS, necessita ter sua atuação e resultados acompanhados (Costa et al., 2013).

Nesse sentido, a avaliação de programas de saúde apresenta-se como uma atividade essencial nas políticas de saúde, por ser uma ferramenta fundamental de feedback, importante não apenas para seus idealizadores, mas para todos os setores sociais envolvidos - formuladores, implementadores e beneficiários (Albuquerque, Melo, Sousa Filho, \& Araújo Neto, 2011). Tarefa que deveria ser realizada em equipe interdisciplinar, por se tratar de um tema transversal a diferentes áreas do conhecimento. Defende-se ainda a participação de psicólogos que sejam capacitados para a construção de instrumentos e medição de crenças, atitudes, valores e representações sociais (Melo, Alchieri, Araújo Neto, \& Melo, 2014). A partir desse processo, é possível, pois, originar mudanças e aperfeiçoar os programas e ações, tornando a avaliação uma etapa imprescindível nas políticas de saúde (Contandriopoulos, 2006; Melo et al., 2014).

Prega-se, portanto, que a avaliação do CEREST é também uma fase indispensável para a manutenção da qualidade das ações desenvolvidas por esses centros, principalmente, porque a literatura é escassa sobre isso. Pretende-se, no presente estudo, realizar uma avaliação do CEREST-CE por meio das crenças de seus usuários e representantes dos sindicatos dos trabalhadores, que são os sujeitos que se encontram no fim da esteira do processo de produção, que veem os resultantes das políticas públicas, que se deparam com seus acertos e seus entraves (Melo, Alchieri, \& Araújo Neto, 2012).

Nesse sentido, a presente pesquisa embasou-se na teoria das crenças da Psicologia Social, partindo do suposto de Rokeach (1981) de que todas as pessoas possuem crenças, que são deduções feitas a respeito de si mesmas e do meio em que estão inseridas, adquiridas pela experiência, no contato direto ou indireto com o objeto de crença. Tais fenômenos não são perceptíveis à observação direta, contudo, de modo indireto, podem ser mensurados, por meio das variáveis dependentes decorrentes dela, tornando possível conceber hipóteses empíricas a partir das crenças (Kruger, 2004). A partir dessa suposição, pode-se deduzir, na presente pesquisa, que esses atores possuem crenças sobre esse programa. Opiniões geradas por meio da vivência dentro desse centro, do contato direto com esse serviço, muitas vezes compartilhadas entre esses sujeitos, que podem intervir e influenciar em suas condutas relacionadas ao CEREST-CE.

Face ao exposto, a presente pesquisa objetiva avaliar o Centro de Referência em Saúde do Trabalhador estadual do Ceará (CEREST-CE) a partir das crenças dos representantes dos sindicatos e usuários. Pretende-se, assim, compreender os fatores facilitadores e complicadores que podem levar ao êxito ou fracasso dessa política, detectar aspectos positivos e, principalmente, identificar possíveis entraves na operacionalização deste programa no Ceará, oferecendo um retorno para a tomada de decisão em busca da melhoria de suas ações.

\section{Método}

\section{Tipo de Estudo}

O presente estudo é uma pesquisa de campo de cunho qualitativo e aplicada (pois pretende dar retorno aos gestores sobre o CEREST cearense). É do tipo exploratório e descritivo, uma vez que busca proporcionar 
maior familiaridade com o problema investigado, visto que este ainda é pouco conhecido e explorado.

\section{Participantes}

Contou-se com uma amostra não probabilística por conveniência composta por oito participantes. Entre eles, havia três representantes de sindicatos integrantes do Conselho Gestor do CEREST-CE (participantes 1 a 3): Participante 1: homem, 40 anos, portuário, representante do Sindicato dos Trabalhadores no Serviço Público Estadual do Ceará (MOVA-SE); Participante 2: mulher, 60 anos, auxiliar de enfermagem, representante do Sindicato dos Empregados em Estabelecimentos de Serviços de Saúde no Estado do Ceará (SINDSAÚDE-CE); Participante 3: homem, 65 anos, técnico em segurança do trabalho, representante do Sindicato dos Técnicos em Segurança do Trabalho do Ceará (SINTEST-CE).

No fim do processo da esteira de produção, contou-se com a participação de cinco usuários do CEREST-CE (participantes 4 a 8): dois usuários atendidos pela equipe de profissionais do centro - Participante 4 (mulher, 50 anos, auxiliar de serviços gerais) e o Participante 5 (homem, 31 anos, pedreiro); dois usuários que participam do Grupo de Apoio a Saúde do Trabalhador (GAST) - Participante 6 (homem, 53 anos, técnico em eletrônica) e o Participante 7 (homem, 54 anos, funcionário público); e um representante de uma empresa usuária (empresa que recebe visita de uma equipe do CEREST-CE para realizar a vigilância em saúde do trabalhador) - Participante 8 (mulher, 58 anos, médica do trabalho).

\section{Instrumento}

Foi utilizado um roteiro de entrevista semiestruturado contendo categorias baseadas na literatura sobre RENAST/CEREST e no discurso ouvido de especialistas da área: (1) ações do CEREST; (2) relação entre sindicato e CEREST; (3) acessibilidade; (4) aspectos positivos e negativos do CEREST; (5) qualidade do atendimento e (6) importância do CEREST para o sindicato.

\section{Procedimento de Análise dos Dados}

Como todas as entrevistas foram gravadas e posteriormente transcritas, a exploração do material de análise foi desenvolvida tomando como referência suas características e semelhanças. Nesse sentido, o suporte metodológico para este estudo, centrou-se na análise de conteúdo com base em Bardin (1977). Esse método tem a finalidade de obter, de forma sistemática, informações que permitam a realização de inferências acerca do objeto de estudo.
Fundamentados nessa forma de analisar, as entrevistas seguiram um meio de interpretação previamente planejado e fragmentado nos seguintes passos: delimitação dos objetivos (elaboração das perguntas do roteiro semiestruturado); constituição do corpus (depois de serem transcritas, cada entrevista retratou um corpus a ser analisado); codificação (definição das Unidades de Contexto Elementar - UCEs); categorização (divisão do conteúdo em classes temáticas, categorias e subcategorias, cálculo das frequências e percentual das UCEs) e; interpretação e inferência (compreensão dos significados e dedução dos resultados finais).

Em seguida, realizou-se uma análise de dados textuais com apoio do software IRAMUTEQ (Interface de $\mathrm{R}$ pour les Analyses Multidimensionnelles de Textes et de Questionnaires). O IRAMUTEQ é um software gratuito e desenvolvido sob a lógica da open source, licenciado por GNU GPL (v2). Ele ancora-se no ambiente estatístico do software $\mathrm{R}$ e na linguagem python. Esse programa informático viabiliza diferentes tipos de análise de dados textuais, desde aquelas bem simples, como a lexicografia básica (cálculo de frequência de palavras), até análises multivariadas (classificação hierárquica descendente, análises de similitude). Ele organiza a distribuição do vocabulário de forma facilmente compreensível e visualmente clara (análise de similitude e nuvem de palavras). Dessa maneira, a partir do IRAMUTEQ, recorreu-se à nuvem de palavras que as agrupa e as organiza graficamente em função da sua frequência. É uma análise lexical mais simples, porém, graficamente bastante interessante, na medida em que possibilita rápida identificação das palavras-chave de um corpus.

\section{Procedimentos Éticos e de Coleta de Dados}

Considerando-se os aspectos éticos referentes a pesquisas envolvendo seres humanos, inicialmente o estudo foi aprovado por comitê de ética em pesquisa sob o parecer $\mathrm{N}^{\circ}$ 1.415.687, de 16 de fevereiro de 2016. Em seguida, iniciou-se o procedimento de coleta de dados, na qual os participantes foram abordados aleatoriamente dentro do CEREST-CE e convidados a participar da pesquisa. Antes do início das entrevistas foram explicitados os objetivos da pesquisa. Em seguida, os participantes foram convidados a assinarem o Termo de Consentimento Livre e Esclarecido (TCLE) e as entrevistas foram realizadas dentro do CEREST-CE, de forma individual, com auxílio de gravador, em sala reservada. Destaca-se que foram seguidas todas as normas para pesquisa com seres humanos, de acordo com a Resolução no 466/12. 


\section{Resultados}

\section{Classe Temática 1 - Ações do CEREST}

Essa classe temática, composta por 719 UCEs, distribuídas em seis categorias, contempla as ações do CEREST sob o ponto de vista dos representantes de sindicatos e dos usuários do CEREST-CE (ver Tabela 1). No que se refere à categoria (1.1) "As ações realizadas pelo CEREST-CE", foram identificadas seis atividades concretizadas pelo CEREST. A primeira delas e a mais valorizada pelos representantes de sindicatos e pelos usuários trata-se do (1.1.1) "Atendimento ao trabalhador", que procuram o centro por demanda espontânea ou encaminhado pelos sindicatos. No CEREST, o trabalhador passa por uma triagem e, em seguida, é encaminhado ao profissional especializado, que faz um levantamento de dados para identificar se a doença é de origem ocupacional.

Quando confirmada a relação com o trabalhado, o usuário é orientado sobre os direitos trabalhistas e previdenciários com a advogada ou encaminhado para consulta com o médico. O centro também atende pessoas com deficiência, que vêm em busca de laudo de homologação de deficiência.

Outra ação relatada pelos representantes de sindicatos e pelos usuários foi a (1.1.2) "Educação em saúde, prevenção e capacitação da rede SUS". O CEREST-CE realiza treinamentos e oferece cursos de formação em saúde do trabalhador para profissionais da rede SUS. Também realiza atividades educativas envolvendo os sindicatos dos trabalhadores, empresas e a sociedade em geral. Outra atividade relatada foi o Grupo de Apoio a Saúde do Trabalhador (GAST) - grupo aberto que se reúne uma vez por mês, formado por trabalhadores atendidos pelo CEREST-CE, tendo por objetivo a troca de experiências e informações que auxiliem a participação ativa dos membros na melhoria da qualidade de vida.

No tocante à (1.1.3) "Vigilância em saúde do trabalhador", o CEREST-CE realiza visita às empresas, acompanhado pelo sindicato que solicitou a fiscalização ou por meio de busca ativa. Nessa visita, são analisadas as condições de trabalho, para constatar se estas estão de acordo com as Normas Regulamentadoras (NR) de Segurança e Saúde do Trabalho e se existe o Serviço Especializado em Engenharia de Segurança e em Medicina do Trabalho (SESMT). Quando necessário, são sugeridas mudanças e realizadas orientações no sentido de prevenir novas ocorrências.

Em relação à (1.2) "Importância das ações do CEREST para os sindicatos", os seus representantes relataram que todas as ações do CEREST-CE são importantes para as entidades sindicais. Inclusive há uma participação do sindicato nos cursos de capacitação, nas palestras sobre prevenção e promoção de saúde e nas visitas as empresas para realizar vigilância em saúde do trabalhador, contudo, essa participação vem diminuindo paulatinamente.

No que concerne a (1.3) "Outras possibilidades de ação do CEREST-CE", os representantes de sindicatos e os usuários sugeriram a oficialização do atendimento ao trabalhador, a ser feita de forma mais organizada, com ampliação do horário de atendimento até o turno da noite. Sugeriram também que fossem contratados profissionais, como psiquiatra e psicólogo para elaborar o nexo causal de doenças mentais relacionadas ao trabalho, bem como advogado, para dar orientações jurídicas e previdenciárias, porque atualmente esse atendimento é prestado por um profissional que trabalha na área administrativa do centro.

Outra possibilidade de ação proposta refere-se à implantação de uma agenda de atividades culturais, como a exibição de filmes com temas voltados para a saúde do trabalhador e direito trabalhista, além de curso de fotografia e vídeo. Por fim, recomendou-se à instituição a notificação dos trabalhadores informais e estatutários no CEREST-CE, pois, atualmente, só são notificados os casos de doenças e acidentes dos trabalhadores celetistas.

Quanto à (1.4) "Qualidade do atendimento no CEREST-CE", os representantes de sindicatos e os usuários possuem a crença de que esta é razoável. Apesar de o usuário ser bem acolhido e alguns relatarem estar satisfeitos com o serviço, eles afirmam que faltam profissionais, como psiquiatra e psicólogo, o que dificulta, na maioria das vezes, o fechamento do nexo causal de transtornos mentais relacionadas ao trabalho. Para os representantes de sindicatos, a dificuldade do estabelecimento da relação entre o quadro clínico e o trabalho ocorre em virtude da falta de conhecimento dos profissionais ou por receio de comprometimento com os patrões das empresas privadas, que se veem prejudicados diante da ocorrência de nexo causal entre problemas de saúde dos trabalhadores e a ocupação laboral.

No que diz respeito às (1.5) "Dificuldades enfrentadas para efetivar as ações do CEREST-CE", as crenças dos representantes de sindicatos e dos usuários revelam que (1.5.1) "A falta de interesse político e problemas de gestão" são questões relevantes e estão interligadas. Atualmente tem relação direta com a inexistência de um gestor efetivo (a gestão é composta 
apenas por um gestor interino) e isso dificulta a definição das responsabilidades, além de impedir a realização das ações propostas pela política. Existe, também, falta de interesse político no sentido de que o programa funcione de acordo com as diretrizes da política de saúde do trabalhador, o que gera problemas de infraestrutura, falta de recursos materiais e humanos.

Outra barreira relatada pelos representantes de sindicatos e pelos usuários foi o (1.5.2) "Repasse de recursos financeiros". O CEREST não possui autonomia financeira, pois o financiamento vem do Governo Federal para o Fundo Nacional de Saúde e são administrados pela Secretaria de Saúde do Estado (SESA). Assim, todos os gastos do CEREST-CE têm que ser autorizados pela Secretaria, dificultando a realização de ações.

Com relação à (1.5.3) "Falta de clareza nas funções do CEREST-CE”, os representantes de sindicato e os usuários relatam que têm dificuldade em saber qual o papel do centro, quais as suas atividades desempenhadas e utilidade. Para eles, o órgão não se mostra de forma clara e existe uma distância entre o que é proposto pela política e o que é realizado efetivamente.

No tocante à (1.5.4) "Falta de profissionais", os representantes dos sindicatos e os usuários expuseram que um dos obstáculos enfrentados é a falta profissionais qualificados (psiquiatra, psicólogo, assistente social e advogado), para prestar assistência integral ao trabalhador usuário do centro, inclusive a assistência jurídica aos trabalhadores e o fechamento de nexo causal de transtornos mentais relacionados ao trabalho. Atualmente, as orientações jurídicas aos trabalhadores usuários são prestadas como favor pela advogada que trabalha no setor administrativo do CEREST-CE.

Outro obstáculo apontado se refere à (1.5.5) "Falta de divulgação do CEREST-CE”. Segundo os usuários, não há divulgação da política de saúde do trabalhador no Ceará, poucas pessoas conhecem o centro e suas ações desenvolvidas, e sugerem maior divulgação para a população, em geral na mídia e nas empresas, por meio da distribuição de folders para os trabalhadores.

Tabela 1

Classe Temática 1 - Açoes do CEREST-CE

Classe Temática 1- Ações do CEREST-CE- 385 UCEs

\begin{tabular}{lll}
\hline Categoria & $f$ & Discurso dos participantes \\
1.1. As ações & 350 & "Nós tivemos apenas uma visita da equipe do CEREST para ver como funcionava o SESMT \\
realizadas pelo & aqui e mostramos que a gente tinha os cuidados com a saúde ocupacional Mas apenas isso, nem \\
CEREST-CE & uma outra ação foi realizada" - Participante 6.
\end{tabular}

1.2. Importância das ações do CEREST

para os sindicatos

\subsection{Outras}

possibilidades de ação do CEREST-CE

\subsection{Qualidade do atendimento no CEREST-CE}

\subsection{Dificuldades enfrentadas para efetivar as ações do CEREST-CE}

$$
\begin{aligned}
& \text { "As ações do Cerest são muito importantes pro sindicato no que diz, respeito à vigilância né, } \\
& \text { no que diz respeito ao acolhimento, no que diz respeito ao médico, fechamento de nexo, é muito } \\
& \text { importante, embora não aconteça" - Participante } 3 .
\end{aligned}
$$

78 "Começar a notificar pessoas que não são celetistas. Pela CLT é obrigatório você abrir uma CAT, mandar pro INSS, agora as pessoas que trabalham por contra própria ou são avulsas ou os próprios estatutários que é também uma das pessoas que o CEREST atende não tem ainda essa visão de doenças do trabalho" - Participante 6.

117 "Por conta dessas muitas carências que tem aqui. Teria tem que melhorar muito.". Participante 6

"No CEREST aqui fui bem recebida, pra receber o laudo, se tem outras coisas ainda não fui informada" - Participante 8.

249 "O poder público muitas vezes toma partido e acaba deixando aquilo lá de forma que não funcione porque não é interessante nê" - Participante 3.

"Cada vez que muda o governo, muda o gestor e lá vai começar tudo do zero de novo" Participante 8. 
Classe Temática 2 - Acessibilidade dos Usuários ao CEREST-CE

Essa classe temática representada por 79 UCEs, distribuídas em três categorias, se refere às crenças dos representantes de sindicatos e dos usuários sobre as formas de acesso ao CEREST-CE (ver Tabela 2). No acesso por meio de (2.1) "Indicação de amigos", os usuários chegam ao Centro por sugestão de pessoas que já foram atendidas ou que trabalham no Centro. Quanto ao acesso por meio da (2.2) "Demanda encaminhada pelos sindicatos", esta se refere aos trabalhadores que chegam aos sindicatos acometidos por alguma doença relacionada ao trabalho em busca de orientação relativa a questões jurídicas e previdenciárias e são encaminhados ao CEREST-CE para serem atendidos pela equipe de profissionais da unidade.

No que se refere ao acesso por meio de (2.3) "Integração com a Rede SUS", os representantes de sindicatos relataram que o CEREST-CE não está sendo referenciado pela Rede de Serviços Sentinela de Vigilância de Violências e Acidentes - Rede VIVA, que visa complementar o sistema de informações existente para a vigilância dessas causas, que visa complementar o sistema de informações existente para a vigilância destas (Gawryszewski et al., 2006). Como consequência, a referência e contrarreferência, ações que compõem um sistema organizador dos aparelhos de saúde, no sentido de permitir o acesso a todo o tipo de serviço oferecido pelo SUS, não tem incluído o CEREST na rede de saúde.

\section{Classe Temática 3 - Importância do CEREST-CE para os Sindicatos}

Essa classe temática, representada por 45 UCEs, retrata o discurso dos representantes de sindicatos sobre a importância do CEREST-CE para os sindicatos (ver Tabela 3). Para eles, o centro, enquanto proposta prescrita por lei é uma ferramenta de suma importância para a saúde do trabalhador em todos os aspectos (pesquisa, educação em saúde do trabalhador, capacitação). Ele assessora o movimento sindical nas demandas de saúde e de ambiente de trabalho. No entanto, alguns representantes o consideram um instrumento nulo, porque não funciona como deveria.

Tabela 2

Classe Temática 2 - Acessibilidade dos Usuários ao CEREST-CE

\begin{tabular}{|c|c|c|}
\hline \multicolumn{3}{|c|}{ Classe Temática 2 - Acessibilidade dos Usuários ao CEREST-CE - 26 UCEs } \\
\hline Categoria & $f$ & Discurso dos participantes \\
\hline $\begin{array}{l}\text { 2.1. Indicação de } \\
\text { amigos }\end{array}$ & 46 & $\begin{array}{l}\text { "Uma amiga já conhecia, me informou sobre o, sobre essa entidade aqui e en precisava, tava tendo } \\
\text { problema de assédio moral e procurei aqui o CEREST". Vim aqui e conversei. Fižeram uma } \\
\text { entrevista aqui comigo né" - Participante } 7 \text {. }\end{array}$ \\
\hline $\begin{array}{l}\text { 2.2. Demanda } \\
\text { encaminhada pelos } \\
\text { sindicatos }\end{array}$ & 16 & $\begin{array}{l}\text { "A forma de acesso do usuário ao CEREST somos nós entidades sindicais que nós orientamos } \\
\text { aos nossos trabalhadores que procure o CEREST" - Participante } 2 .\end{array}$ \\
\hline $\begin{array}{l}\text { 2.3. Referência e } \\
\text { contra referência } \\
\text { (Integração com a } \\
\text { Rede SUS) }\end{array}$ & 17 & $\begin{array}{l}\text { "Eu sei que no SUS existem os hospitais que fazem parte da rede sentinela e que essa rede devia } \\
\text { identifica e encaminhar esses trabalhadores adoecidos pro CEREST mas, efetivamente me parece } \\
\text { que isso não acontece né. Essa rede sentinela, ela não atua da forma como deveria nos casos é de } \\
\text { adoecimento provocado pelo trabalho" - Participante } 1 \text {. }\end{array}$ \\
\hline
\end{tabular}

Tabela 3

Classe Temática 3 - Importância do CEREST-CE para os Sindicatos

Classe temática 3 - Importância do CEREST-CE para os Sindicatos - 45 UCEs

$f \quad$ Discurso dos participantes

45 "Então, pra mim, enquanto dirigente sindical, o CEREST é um instrumento nulo. E ele é importantíssimo né. O

CEREST tem um significado muito forte que a gente precisa que funcione" - Participante 3. 
Por fim, foi realizada a análise das entrevistas realizadas com representantes de sindicatos e usuários do CEREST-CE por meio do software IRAMUTEQ. A Figura 1 foi elaborada a partir da nuvem de palavras, técnica que agrupa as palavras e as organiza graficamente em função de sua frequência.

Observa-se que as palavras que mais apareceram nas falas dos participantes fazem referência aos principais tópicos abordados nas classes temáticas apresentadas no decorrer deste estudo. Destacam-se: CEREST, trabalhador, saúde, trabalho, sindicato, conhecimento, direito, política, atendimento, referência, vigilância. Reforça, portanto, que, para os representantes dos sindicatos e usuários do CEREST, é importante a figura do trabalhador, assim como as ações voltadas para sua saúde. Restou, evidenciada ainda, a importância dos sindicatos e do referenciamento para acesso do usuário.

\section{Discussão}

Este estudo permitiu compreender a dinâmica de funcionamento do CEREST-CE em diversos aspectos, por meio da análise das crenças dos representantes de sindicatos dos trabalhadores e usuários do CEREST-CE. No que se refere às ações realizadas pelo centro, evidenciou-se que o atendimento ao trabalhador é a ação mais valorizada por todos os participantes. Nesses atendimentos os trabalhadores, buscam orientação sobre direitos trabalhistas e previdenciários ou laudo para homologação de deficiência. Com a instituição da RENAST, em 2002, pela portaria n. ${ }^{\circ}$ 1.679, os CERESTs deixaram de ser porta de entrada do sistema, constituindo-se como centro articulador e organizador no seu território de abrangência, das ações intra e intersetoriais de Saúde do Trabalhador. A assistência à saúde do trabalhador passou a ser de responsabilidade

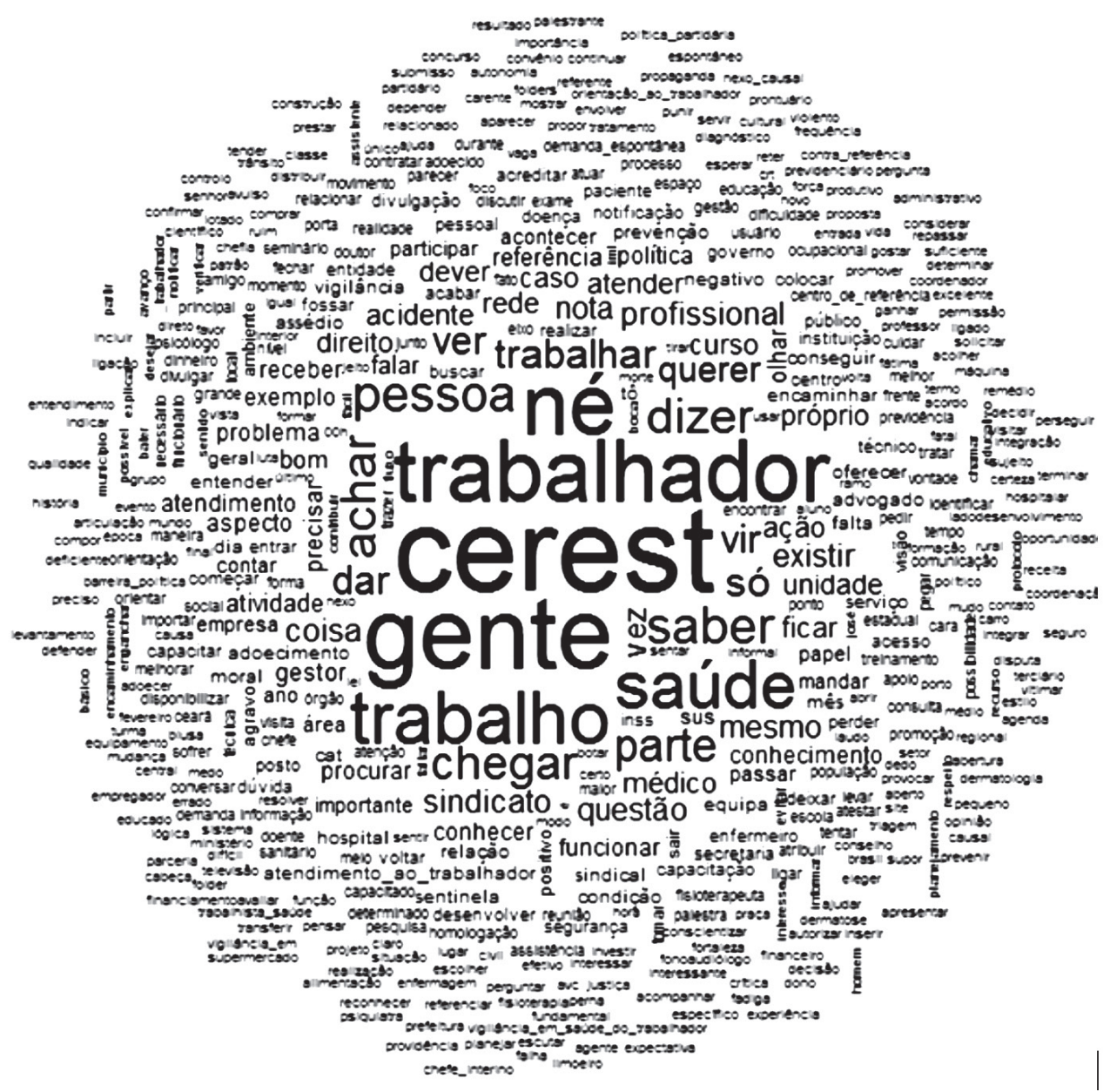

Figura 1. Nuvem de palavras mais frequentes nas falas dos participantes. 
da Atenção Primária, sendo realizada, mais especificamente, no âmbito da Estratégia de Saúde da Família (Lourenço \& Lacaz, 2013; MS, 2005).

Dias e Silva (2013) destacam que o acolhimento aos trabalhadores e a assistência ao usuário trabalhador são possibilidades concretas de ações de saúde que devem ser desenvolvidas na Atenção Básica, por meio da ESF. No entanto, para que isso se concretize, não basta acrescentar mais atribuições às já sobrecarregadas equipes. É importante que estas sejam redefinidas, que as equipes sejam capacitadas e garantidas a referência e contrarreferência do sistema (Quilião et al., 2013).

No discurso dos participantes, observou-se também uma valorização do grupo de apoio (GAST) e sua importância no apoio ao trabalhador adoecido, em detrimento das demais ações de educação em saúde, prevenção e capacitação, ainda pouco valorizadas por esses participantes, e até desconhecidas por alguns. É função do CEREST estadual participar do Polo Estadual de Educação Permanente, apontando as necessidades de capacitação e formação em saúde do trabalhador, no âmbito estadual, assim como de colaborações na elaboração de programas de formação, especialização e qualificação de profissionais de saúde para execução das ações nessa área (MS, 2012; Medeiros et al., 2013).

Destaca-se também a falta de conhecimento dos usuários sobre a vigilância em saúde do trabalhador. Essa ação foi citada apenas pelos representantes de sindicatos, que solicitam ao CEREST-CE as intervenções nas empresas e acompanham as visitas da equipe do Centro às empresas. Segundo Machado et al. (2013), a vigilância em saúde do trabalhador busca intervir na transformação do trabalho, no sentido da promoção da saúde, por meio de uma relação mútua entre profissionais de saúde e trabalhadores. Para o planejamento das ações, é essencial que se faça uso de dados epidemiológicos, entre eles, os seguintes: verificação a partir de eventos notificados, dos casos de risco identificados ou de casos índice; que se faça busca ativa de eventos; que sejam aplicados inquéritos epidemiológicos e que se parta de episódios sentinelas para a investigação (Costa et al., 2013; MS, 2005). Os resultados do estudo evidenciaram que, apesar dos esforços da equipe do centro em desenvolver as ações de saúde do trabalhador, observa-se o favorecimento da assistência em detrimento das ações de vigilância e, até mesmo, prevenção e promoção de saúde do trabalhador, afetando a integralidade das ações oferecidas pelo centro.
No que se refere a outras possibilidades de ações, os usuários considerados consumidores desse produto mostram-se satisfeitos com os serviços e o atendimento oferecido pelo CEREST-CE. Propuseram apenas que seja implantada uma assistência completa e organizada, demonstrando falta de conhecimento sobre a política de saúde do trabalhador e suas atribuições de prevenção, promoção e vigilância em saúde do trabalhador. Por outro lado, contempla-se que, para além da falta de conhecimento dos usuários sobre o funcionamento do CEREST, é relevante destacar que a busca por essa assistência no Centro ocorre também pela negligência do Instituto Nacional do Seguro Social (INSS), que não oferece um serviço eficaz de reabilitação do trabalhador (Souza \& Virgens, 2013). Já os representantes de sindicatos avaliam que o atendimento no CEREST-CE é razoável, devido à falta de resolutividade, e sugeriram a contratação de profissionais para uma assistência integral ao trabalhador adoecido.

O presente estudo revelou ainda, por meio dos discursos dos participantes, uma sucessão de entraves para a operacionalização da Política de Saúde do Trabalhador no Ceará. Os representantes de sindicatos e usuários elencam uma série de dificuldades, a começar por problemas políticos e de gestão, caracterizados pela falta de continuidade na gestão, ou a política de "esvaziar gavetas" do CEREST-CE, pois, a cada troca de secretário de saúde, troca-se o coordenador do Centro, o que impede, não só a concretização dos objetivos, mas a fluidez das ações em saúde do trabalhador. Isso porque os indicados para o cargo não necessariamente incorporam a proposta institucional do programa, ou seja, o mero assistencialismo sobrepujou, cedendo espaço para ações médico/curativa/assistencialista (Leão \& Castro, 2013). Assim sendo, é possível perceber certa subordinação da Política de Saúde do Trabalhador aos interesses políticos partidários, o que acaba por impossibilitar, por vezes, a trajetória de uma saúde do trabalhador mais eficaz no Ceará.

Outra barreira importante apontada por esses atores foi a falta de clareza do papel do CEREST. Essa obscuridade tem raízes na legislação que instituiu e orienta a RENAST. Ela retirou o destaque dos CERESTs nas ações assistenciais e, paradoxalmente, recomendou uma equipe mínima para o centro, sem esclarecer as funções e as incumbências de cada um, tornando difícil a composição da equipe e o processo de capacitação desses profissionais. Contempla-se ainda que a composição da equipe também é dificultada já no próprio processo de formação dos profissionais de 
saúde durante a graduação, pois a Segurança e Saúde no Trabalho (SST) não são incorporadas nas ações formativas dos cursos de graduação, conforme previsto na Portaria Interministerial MPS/MS/MT n. 800 (2005). Como consequência, a falta de clareza sobre o papel do centro e a defasagem entre trabalho prescrito e trabalho real pode estar dificultando a construção de programas de saúde do trabalhador (Daldon, 2012).

Outra dificuldade a ser superada, segundo esses atores, é a ausência de autonomia financeira do CEREST e a burocracia dos processos de contratação de profissionais, aquisição de equipamentos, planejamento de ações educativas, capacitação de profissionais e de vigilância em saúde do trabalhador que, na sua maioria, são muito lentos e até inviáveis. Fato agravado no CEREST-CE que, diferente de outros centros brasileiros, a maioria (Leão \& Vasconcellos, 2001) não possui parcerias com as universidades e sindicatos para a capacitação de seus profissionais.

Conforme o MS (2006), os recursos devem ser repassados do Fundo Nacional de Saúde para os Fundos de Saúde dos Estados, do Distrito Federal e dos Municípios, no bloco de gestão do SUS e no bloco de financiamento da média e alta complexidade, conforme o caso, e serão aplicados pelas Secretarias de Saúde e fiscalizados pelo Conselho de Saúde, o que dificulta a realização de ações.

Neste estudo, constatou-se também que a acessibilidade dos usuários ao CEREST-CE por meio de indicação de amigos ou encaminhado pelos sindicatos é considerada de boa qualidade por todos os envolvidos, uma vez que todo trabalhador que chega ao centro é atendido. Contudo, para os representantes dos sindicatos, há a necessidade de inclusão do CEREST no sistema de referência e contra referência do SUS, para garantir a integralidade da assistência aos trabalhadores portadores de agravos ou doenças relacionadas ao trabalho. A atenção básica é considerada a "porta de entrada" do serviço de saúde, pois, a depender da necessidade do usuário, ele é referenciado para uma unidade com maior complexidade (unidade de referência). Sendo que, após o atendimento, o mesmo usuário deve ser contra referenciado à unidade de atenção básica, ou seja, a unidade especializada volta a encaminhar o paciente à unidade de atenção básica, para que ele continue a ser assistido (MS, 2012; Quilião et al., 2013). Faz-se, portanto, necessária a integração dos serviços de saúde do trabalhador entre a atenção básica e o CEREST-CE (Dias, Rigotto, Augusto, Cancio, \& Hoefel, 2009).
Destaca-se também que os representantes de sindicatos consideram o CEREST-CE um instrumento de suma importância para a saúde do trabalhador. No entanto, avaliam-no como um instrumento nulo, pois ele não desempenha o papel a que se propõe, previsto na legislação. Apesar da importância da participação dos sindicatos no planejamento e construção das ações do CEREST-CE, essa relação encontra-se fragilizada no contexto cearense, e tem diminuído com o passar do tempo, por divergências de interesses sociais e políticos.

Dados os entraves identificados, destaca-se o esforço e a vontade da equipe do CEREST-CE que, em meio a tantas dificuldades, desenvolve projetos de capacitação na rede SUS e realiza campanhas de educação em saúde e seminários para disseminação de informações à sociedade sobre o tema saúde do trabalhador. É importante ressaltar, também, a assistência prestada aos trabalhadores com orientações sobre os direitos trabalhistas e previdenciários e a emissão de atestado médico para homologação, ainda que não seja uma atribuição prevista nas diretrizes da política de saúde do trabalhador.

\section{Considerações Finais}

A partir da análise das crenças dos representantes dos sindicatos e usuários, puderam-se descortinar as barreiras e entraves que dificultam a operacionalização da política de saúde do trabalhador pelo CEREST-CE, contemplando a importância da manutenção da avaliação de suas ações, como forma de proporcionar feedback aos gestores sobre a qualidade de seus serviços, contribuindo, dessa forma, para a tomada de decisões e o aprimoramento do serviço.

Pode-se constatar que prevalece um distanciamento entre as concepções teóricas e o CEREST concreto, tal como ocorre na realidade. Propõe-se que o problema da saúde deixe de ser procurado nos papéis e nas formulações teóricas da política, e sim na operacionalização, no cotidiano do repasse de verbas, na contratação e capacitação dos profissionais e na divulgação da política. Passou-se 14 anos desde a instituição da RENAST e 12 anos desde criação do Centro Manuela Jacaré. No entanto, muitos entraves ainda são encontrados que dificultam a oferta de serviços pelo CEREST-CE. Convoca-se, portanto, a transpassar o discurso sobre o padrão teórico ideal e debater seus entraves reais. É chegada a hora de lutar pela sua melhoria, evidenciando a necessidade crescente de 
fiscalização e de cobrança de resultado das políticas públicas e avaliação do retorno do investimento dado a esses programas, transformados, efetivamente, em políticas de saúde eficientes e eficazes.

Faz-se necessário tratar "saúde" e "trabalho" como duas facetas integradas, indissociáveis. Devem estar articuladas na Rede Intrassetorial, nos diferentes níveis de Atenção do Sistema Único de Saúde (com diálogo direto entre a Estratégia Saúde da Família e o Centro de Referência em Saúde do Trabalhador). Igualmente articuladas na Rede Intrassetorial, sob responsabilidade do SUS, mas também do Ministério Público do Trabalho (MPT) e Instituto Nacional do Seguro Social (INSS), por meio do serviço de perícia e reabilitação do trabalhador e das universidades, pela promoção de capacitação dos trabalhadores e pela oferta de serviços de extensão que ampliem o atendimento da população. Assim, pode ser possível trazer a saúde como direito de fato ao trabalhador.

Algumas limitações podem, entretanto, ser apontadas na execução da presente pesquisa, especialmente no que concerne aos participantes: embora tenha sido proposta a participação de mais usuários do CEREST-CE, no momento da realização da pesquisa havia poucos usuários, o que diminuiu o tamanho da amostra. Sugere-se, portanto, o seguimento de pesquisas a serem executadas com mais usuários e com outros atores sociais envolvidos com o CEREST-CE, inclusive em outros estados brasileiros, pois ainda existe muita história a ser investigada sobre os programas e serviços de saúde do trabalhador no Brasil. Igualmente, cabe ainda reiterar a importância de se priorizar, de forma sistemática, processos de avaliação da RENAST e dos CERESTs, na medida em que indicam a dinâmica própria de cada região no empenho com as demandas de saúde do trabalhador.

\section{Referências}

Albuquerque, F. J. B., Melo, C. F., Sousa Filho, F. E., \& Araújo Neto, J. L. (2011). Avaliação da estratégia saúde da família a partir das crenças dos profissionais. Estudos de Psicologia (Campinas), 28(3), 363-370. doi: 10.1590/S0103-166X2011000300008

Balista, S. R. R., Santiago, S. M., \& Corrêa Filho, H. R. (2011). A descentralização da vigilância da saúde do trabalhador no município de Campinas, São Paulo, Brasil: Uma avaliação do processo. Cadernos de Saúde Pública, 27(4), 58-70. doi: 10.1590/ S0102-311X2011000400015.
Bardin, L. (1977). Análise de conteúdo. Lisboa: Edições 70.

Contandriopoulos, A. P. (2006). Avaliando a institucionalização da avaliação. Ciência \& Saúde Coletiva, 11(3), 705-711. doi: 10.1590/S1413-81232006000300017.

Costa D., Lacaz, F. A. C., Jackson Filho, J. M., \& Vilela, R. A. G. (2013). Saúde do trabalhador no SUS: Desafios para uma política pública. Revista brasileira de saúde ocupacional, 38(127), 11-30. doi: 10.1590/ S0303-76572013000100003

Daldon, M. T. B. (2012). Processo de trabalho dos profissionais de saúde em vigilancia em saúde do trabalbador (Tese de doutorado). Universidade de São Paulo. São Paulo, Brasil. Recuperado de http:// www.teses.usp.br/teses/disponível/5/5163/ tde-18012013-104545.

Dias, E. C., Rigotto, R. M, Augusto. L. G. S., Cancio, J., \& Hoefel, M. G. L.. (2009). Saúde ambiental e saúde do trabalhador na atenção primária à saúde, no SUS: Oportunidades e desafios. Ciência \& Saúde Coletiva, 14(6), 2061-2070. doi: 10.1590/ S1413-81232009000600013.

Dias, E. C., \& Silva T. L. (2013). Contribuições da atenção primária em saúde para a implementação da política nacional de saúde e segurança no trabalho (PNSST). Revista Brasileira de Saúde Ocupacional, 38(127), 3143. doi: 10.1590/S0303-76572013000100007

Gawryszewski, V. P., Silva, M. M. A., Malta, D. C., Mascarenhas, M. D. M., Costa, V. C., Matos, S. G., Moraes Neto, O. L., Monteiro, R. A., Carvalho, C. G.l, \& Magalhães, M. L. (2006). A proposta da rede de serviços sentinela como estratégia da vigilância de violências e acidentes. Ciência \& Saúde Coletiva, 11(Suppl), 1269-1278. doi: 10.1590/ S1413-81232006000500016

Jacques, C. C., Milanez, B., \& Mattos, R. C. O. (2012). Indicadores para centros de referência em saúde do trabalhador: Proposição de um sistema de acompanhamento de serviços de saúde. Ciência e saúde coletiva, 17(2), 369-378. doi: 10.1590/ S1413-81232012000200011.

Kruger, H. (2004). Crenças compartilhadas, preconceitos e discriminações. Em Sociedade Brasileira de Psioclogia [SBP] (Eds.), XXXIV Reunião Anual da Sociedade Brasileira de Psicologia, Ribeirão Preto, SP.

Lacaz, F. A. C., Trapé, A., Soares, C. B., \& Santos, A. P. L. (2013). Estratégia saúde da

Psico-USF, Bragança Paulista, v. 23, n. 1, p. 127-138, jan./mar. 2018 
família e saúde do trabalhador: Um diálogo possível? Interface (Botucatu), 17(44), 75-87. doi: 10.1590/ S1414-32832013000100007.

Leão, L. H. C., \& Castro, A. C. (2013). Políticas públicas de saúde do trabalhador: Análise da implantação de dispositivos de institucionalização em uma cidade brasileira. Ciência \& Saúde Coletiva, 18(3), 769-778. doi: 10.1590/S1413-81232013000300023.

Leão, L. H. C., \& Vasconcellos, L. C. F. (2011). Rede nacional de atenção integral à saúde do trabalhador (Renast): Reflexões sobre a estrutura de rede. Epidemiologia e Serviços de Saúde, 20(1), 85-100. doi: 10.5123/S1679-49742011000100010

Lourenço, E. A. S., \& Lacaz, F. A. C. (2013). Os desafios para a implantação da política de saúde do trabalhador no SUS: O caso da região de Franca-SP. Revista Brasileira de Saúde Ocupacional, 38(127), 44-56. doi: 10.1590/S0303-76572013000100008.

Machado, J. M. H., Campos, A., Ferrite, S., Peres, M. C., Galdino, A., Neto, R. V., Veiga, R. M. P., \& Santos, A. P. L. (2013). Situação da rede nacional de atenção integral em saúde do trabalhador (RENAST) no Brasil, 2008-2009. Revista Brasileira de Saúde Ocupacional, 38(128), 243-256. doi: 10.1590/ S0303-76572013000200012.

Medeiros, M. A. T., Sarleno, V. L., Silvestre, M. P., \& Magalhães, L. V. (2013). Política de saúde do trabalhador: Revisitando o caso do centro de referência em saúde do trabalhador de Campinas. Revista Brasileira de Saúde Ocupacional, 38(127), 81-91. doi: 10.1590/S0303-7657201300 0100011.

Melo, C. F., Alchieri, J. C., \& Araújo Neto, J. L. (2012). Sistema Único de Saúde: Uma avaliação realizada em Natal, Rio Grande do Norte, Brasil. Psico-USF, 17(1), 63-72. doi: 10.1590/ S1413-82712012000100008.

Melo, C. F., Alchieri, J. C., Araújo Neto, J. L., \& Melo, F. A. F. (2014). Desenvolvimento de instrumentais para avaliação da estratégia saúde da família em Natal. Psicologia: Reflexão e Crítica, 27(2), 219-227. doi: 10.1590/1678-7153.201427202.

Ministério da Saúde [MS]. (2002). Portaria n 1.679, de 19 de setembro de 2002. Dispõe sobre a estruturação da Rede Nacional de Atenção Integral à Saúde do Trabalhador no SUS. Brasília: Autor. Disponível: http:/ / ftp.medicina.ufmg.br/osat/legislacao/Portaria_1679_12092014.pdf
Ministério da Saúde [MS]. (2005). Portaria n $n^{\circ} 2.437$ de 07 de dezembro de 2005. Dispõe sobre a ampliação e o fortalecimento da Rede Nacional de Atenção Integral à Saúde do Trabalhador - RENAST no Sistema Único de Saúde - SUS. Brasília: Autor. Disponível: http://www.cerest.piracicaba.sp.gov. br/site/images/ Portarian2437-RENAST.pdf.

Ministério da Saúde [MS]. (2005). Portaria Interministerial MPS/MS/MT no 800 de 3 de maio de 2005. Publica o texto base da Minuta de Política Nacional de Segurança e Saúde do Trabalho. Brasília: Autor. Recuperado de http://ftp.medicina.ufmg.br/osat/ legislacao/Portaria_800_22092014.pdf.

Ministério da Saúde [MS]. (2006). Rede Nacional de Atenção Integral à saúde do Trabalhador (Manual de Gestão e Gerenciamento) (1 ${ }^{\text {a }}$ edição). Brasilia: Autor. Recuperado de http://bvsms.saude.gov.br/bvs/ publicacoes/ManualRenast06.pdf

Ministério da Saúde [MS]. (2009). Portaria n 2.728, de 11 de agosto de 2009. Dispõe sobre a Rede Nacional de Atenção Integral à Saúde do Trabalhador (RENAST). Brasília: Autor. Recuperado de http:// saude.es.gov.br/Media/sesa/CEREST/site $\% 20$ \%20portaria_renast_2728.pdf

Ministério da Saúde [MS]. (2012). Portaria n 1.823, de 23 de agosto de 2012. Institui Política Nacional de Saúde do Trabalhador e da Trabalhadora. Brasília: Autor. Recuperado de http://bvsms.saude.gov.br/bvs/ saudelegis/gm/2012/prt1823_23_08_2012.html

Ministério da Saúde [MS]. (2016). Portaria n 205, de 17 de fevereiro de 2016. Define a lista nacional de doenças e agravos. Brasília: Autor. Disponível: https:// central3.to.gov.br/arquivo/296834/.

Oliveira, A. K. L. (2013). Assédio moral no cenário do trabaIho flexivel: Desafios da vigilância em Saúde do Trabalhador no Ceará (Dissertação de mestrado). Universidade Estadual do Ceará, Fortaleza, Brasil. Recuperado de http://www.uece.br/politicasuece/dmdocuments/Ana_Karina_Loiola.pdf

Quilião, P. L., Fassa, A. G., \& Restrepo, M. C. (2013). Processo de implantação de um centro regional de referência em saúde do trabalhador no Rio Grande do Sul. Revista Brasileira de saúde ocupacional, 38(128), 257-267. doi: 10.1590/S0303-76572013000200013.

Rokeach, M. (1981). Crenças, atitudes e valores. Rio de Janeiro: Interciência. 
Souza, T. S., \& Virgens, L. S. (2013). Saúde do trabalhador na atenção básica: Interfaces e desafios. Revista Brasileira de Saúde Ocupacional, 38(128), 292-301. doi: 10.1590/S0303-76572013000200016.
Recebido em: 25-01-2017

Reformulado em: 22-05-2017; 02-06-2017

Aprovado em: 06-06-2017

Sobre as autoras:

Suzete Rodrigues Leônidas é fisioterapeuta e mestre em Psicologia pela Universidade de Fortaleza (UNIFOR). E-mail: suzeterl@gmail.com

Cynthia de Freitas Melo é psicóloga e doutora em Psicologia (UFRN), professora do Programa de Pós-Graduação em Psicologia da Universidade de Fortaleza (UNIFOR) e coordenadora do Laboratório de Estudos e Práticas em Psicologia e Saúde (LEPP - Saúde).

E-mail:cf.melo@yahoo.com.br

Contato com as autoras:

Cynthia de Freitas Melo

Av. Washington Soares, 1321, Bloco N, Sala N13

Bairro Edson Queiroz

Fortaleza-CE, Brasil

CEP: 60.811-341 